Supporting Information

for

\title{
Air-stable n-Type Semiconductor: \\ Core-perfluoroalkylated Perylene Bisimides
}

\author{
Yan Li, Lin Tan, Zhaohui Wang,* \\ Hualei Qian, Yubai Shi and Wenping Hu \\ Beijing National Laboratory for Molecular Sciences, Key Laboratory of Organic Solids, \\ Institute of Chemistry, Chinese Academy of Sciences, \\ Beijing 100080, P.R. China \\ wangzhaohui@iccas.ac.cn
}

\section{Contents}

$\begin{array}{ll}\text { Experimental } & \mathrm{S} 2\end{array}$

$\begin{array}{ll}\text { a. General Data and Procedure. } & \text { S2 }\end{array}$

$\begin{array}{ll}\text { b. Synthesis of } 2 \mathrm{a} & \mathrm{S} 2\end{array}$

$\begin{array}{ll}\text { c. Synthesis of } 3 \mathrm{a} & \mathrm{S} 3\end{array}$

d. Synthesis of $2 b \quad$ S3

e. Synthesis of $2 c \quad$ S3

Molecular structure and Crystal packing $\quad$ S4

$\begin{array}{ll}\text { a. Molecular structure and Crystal packing of } 2 b & \text { S4 }\end{array}$

b. Molecular structure and Crystal packing of 2c S5

References $\quad$ S6

$\begin{array}{ll}\text { Spectral Data } & \text { S7 }\end{array}$ 


\section{Experimental}

\section{a. General Data.}

${ }^{1} \mathrm{H}$ and ${ }^{13} \mathrm{C}$ NMR spectra were recorded with a Bruker ADVANCE 400 NMR spectrometer in $\mathrm{CDCl}_{3}$ using TMS as internal standard. Chemical shifts $(\delta)$ are expressed in ppm downfield from tetramethylsilane using the residual non-deuterated solvent as internal standard $\left(\mathrm{CDCl}_{3}:{ }^{1} \mathrm{H} 7.26\right.$ ppm, ${ }^{13} \mathrm{C} 77.0 \mathrm{ppm}$ ). Mass spectra (MALDI-TOF-MS) were determined on a Bruker BIFLEX III Mass Spectrometer. UV-Vis spectra were recorded with Hitachi (model U-3010) UV-Vis spectrophotometer in a 1-cm quartz cell. All fluorescence spectra are corrected. The fluorescence quantum yields were determined by optical dilute method $^{1}(\mathrm{~A}<0.05)$ using $N, N^{\prime}$-di(2,6-diisopropylphenyl)-perylene-3,4:9,10-tetracarboxylic acid bisimide $\left(\varphi_{\mathrm{fl}}=1.00 \mathrm{in}\right.$ chloroform $)^{2}$ as reference. Cyclic voltammetry (CV) was performed with a Zahner IM6e electrochemical workstation using glassy carbon discs as the working electrode, Pt wire as the counter electrode, $\mathrm{Ag} / \mathrm{AgCl}$ electrode as the reference electrode, and ferrocene/ferrocenium as an internal potential marker. $0.1 \mathrm{M}$ tetrabutylammonium hexafluorophosphate $\left(\mathrm{TBAPF}_{6}\right)$ dissolved in $\mathrm{CH}_{2} \mathrm{Cl}_{2}$ was employed as the supporting electrolyte. $\mathrm{CH}_{2} \mathrm{Cl}_{2}$ was freshly distilled prior to use. All chemicals were purchased from commercial suppliers and used without further purification unless otherwise specified. Starting materials dibromoperylene bisimides 1a, b, c were synthesized according to literature. ${ }^{3}$ DMSO was freshly distilled from $\mathrm{CaH}_{2}$.

\section{b. General Copper-Mediated Cross-Coupling Procedure.}

A suspension of $\mathrm{Cu}$ bronze (10 equiv) in dry DMSO $(0.5-1 \mathrm{M})$ was heated at $110-130{ }^{\circ} \mathrm{C}$ for 15-30 min under nitrogen. Perfluoroalkyl iodide (5 equiv) was added dropwise in such a way as to keep the temperature below $135{ }^{\circ} \mathrm{C}$. After $45-60$ min, a solution of 1 (1 equiv) in the same solvent (0.1-0.5 M) was added dropwise, and the reaction mixture was stirred at $110-130{ }^{\circ} \mathrm{C}$ for $8 \mathrm{~h}$. After cooling down to room temperature, the reaction mixture was poured into water and the product was extracted with diethyl ether. The aqueous layer was extracted with diethyl ether once more and the two organic phases were combined, washed with water, and dried over $\mathrm{MgSO}_{4}$. The crude product was purified by silica gel chromatography.

\section{c. Synthesis of $N, N$ '-Di(2,6-diisopropylphenyl)-1,7-dinonafluorobutylperylene $-3,4: 9,10-$ tetracarboxylic acid bisimide (2a).}

According to general procedure, the suspension of $740 \mathrm{mg} \mathrm{Cu}$ bronze $(11.6 \mathrm{mmol})$ in dry $20 \mathrm{ml}$ DMSO was heated at $125^{\circ} \mathrm{C}$ for $15 \mathrm{~min}$ under nitrogen. $2 \mathrm{~g}$ Iodononafluorobutane $(5.8 \mathrm{mmol})$ was added dropwise. After $45 \mathrm{~min}$, a solution of $1 \mathrm{~g} 1 \mathrm{a}(1.2 \mathrm{mmol})$ in $5 \mathrm{ml}$ DMSO was added dropwise, and the reaction mixture was stirred at $125{ }^{\circ} \mathrm{C}$ for $8 \mathrm{~h}$ yielded after column chromatography $\left(\mathrm{CH}_{2} \mathrm{Cl}_{2}: n\right.$-hexane $\left.=1: 2\right) 90 \%$ of $\mathbf{2 a}$ as an orange solid.

${ }^{1} \mathrm{H}$ NMR $\left(\mathrm{CDCl}_{3}, 400 \mathrm{MHz}, 298 \mathrm{~K}\right): \delta=8.98(\mathrm{~s}, 2 \mathrm{H}$, perylene-H), $8.76(\mathrm{~d}, 2 \mathrm{H}, \mathrm{J}=8 \mathrm{~Hz}$, perylene-H), 8.33 (s, 2H, perylene-H), 7.47 (t, $2 \mathrm{H}, \mathrm{J}=8 \mathrm{~Hz}$, phenyl- $\mathrm{H}), 7.30(\mathrm{~d}, 4 \mathrm{H}, \mathrm{J}=8 \mathrm{~Hz}$, phenyl-H), 2.69 (m, 4H, isopropyl-H), 1.13 (m, 24H, isopropyl-H); ${ }^{13} \mathrm{C} \mathrm{NMR}\left(\mathrm{CDCl}_{3}, 100 \mathrm{MHz}\right.$, $298 \mathrm{~K}): \delta=162.8,162.4,145.6,135.7,132.7,132.5,131.7,131.3,130.0,129.9,128.7,128.6$, 127.3, 127.0, 126.8, 124.2, 123.6, 123.4, 118.8, 116.9, 115.9, 29.3, 24.0. MS (MALDI-TOF): m/z $\left(M^{-}\right)=1147.5$ (calcd for $\mathrm{C}_{56} \mathrm{H}_{40} \mathrm{~F}_{18} \mathrm{~N}_{2} \mathrm{O}_{4}$ : 1146.9); elemental analysis: calcd (\%) for $\mathrm{C}_{56} \mathrm{H}_{40} \mathrm{~F}_{18} \mathrm{~N}_{2} \mathrm{O}_{4}$ : C 58.65, H 3.52, N 2.44; found C 58.59, H 3.53, N 2.57; UV/Vis $\left(\mathrm{CHCl}_{3}\right): \lambda_{\max }(\varepsilon)=510$ (55337), 
$477 \mathrm{~nm}\left(38758 \mathrm{M}^{-1} \mathrm{~cm}^{-1}\right)$; fluorescence $\left(\mathrm{CHCl}_{3}\right): \lambda_{\max }=526 \mathrm{~nm}$, fluorescence quantum yield $\Phi_{\mathrm{fl}}=$ 0.92; $\mathrm{CV}\left(\mathrm{CH}_{2} \mathrm{Cl}_{2}, 0.1 \mathrm{M}\right.$ TBAHFP, vs. $\left.\mathrm{Fc} / \mathrm{Fc}^{+}\right): \mathrm{E}_{1 / 2}(\mathrm{PBI} / \mathrm{PBI})=-0.67 \mathrm{~V}, \mathrm{E}_{1 / 2}\left(\mathrm{PBI}^{-} / \mathrm{PBI}^{2-}\right)=$ $-0.99 \mathrm{~V}$.

\section{d. Synthesis of $N, N^{\prime}$-Di(2,6-diisopropylphenyl)-1,7-diheptadecafluorooctyl perylene-3,4:9,10-tetracarboxylic acid bisimide (3a).}

According to general procedure, the suspension of $740 \mathrm{mg} \mathrm{Cu}$ bronze $(11.6 \mathrm{mmol})$ in dry $20 \mathrm{ml}$ DMSO was heated at $125{ }^{\circ} \mathrm{C}$ for $15 \mathrm{~min}$ under nitrogen. $3.1 \mathrm{~g}$ 1-Iodoperfluorooctane $(5.8 \mathrm{mmol})$ was added dropwise. After $45 \mathrm{~min}$, a solution of $1 \mathrm{~g}$ 1a $(1.2 \mathrm{mmol})$ in $5 \mathrm{ml}$ DMSO was added dropwise, and the reaction mixture was stirred at $125{ }^{\circ} \mathrm{C}$ for $8 \mathrm{~h}$ yielded after column chromatography $\left(\mathrm{CH}_{2} \mathrm{Cl}_{2}: n\right.$-hexane $\left.=1: 2\right) 85 \%$ of $3 \mathbf{a}$ as an orange solid.

${ }^{1} \mathrm{H}$ NMR $\left(\mathrm{CDCl}_{3}, 400 \mathrm{MHz}, 298 \mathrm{~K}\right): \delta=8.98(\mathrm{~s}, 2 \mathrm{H}$, perylene-H), $8.76(\mathrm{~d}, 2 \mathrm{H}, \mathrm{J}=8 \mathrm{~Hz}$, perylene-H), $8.33(\mathrm{~s}, 2 \mathrm{H}$, perylene-H), $7.47(\mathrm{t}, 2 \mathrm{H}, \mathrm{J}=8 \mathrm{~Hz}$, phenyl- $\mathrm{H}), 7.31(\mathrm{~d}, 4 \mathrm{H}, \mathrm{J}=8 \mathrm{~Hz}$, phenyl-H), 2.68 (m, 4H, isopropyl-H), $1.13(\mathrm{~m}, 24 \mathrm{H}$, isopropyl- $\mathrm{H}) ;{ }^{13} \mathrm{C} \mathrm{NMR}\left(\mathrm{CDCl}_{3}, 100 \mathrm{MHz}\right.$, $298 \mathrm{~K}): \delta=162.8,162.4,145.7,135.7,132.7,131.7,131.4,130.0,129.9,128.7,128.6,127.2$, 124.3, 123.7, 123.4, 31.6, 29.3, 29.1, 24.0, 22.6, 14.1. MS (MALDI-TOF): $\mathrm{m} / \mathrm{z}\left(\mathrm{M}^{-}\right)=1546.7$ (calcd for $\mathrm{C}_{64} \mathrm{H}_{40} \mathrm{~F}_{34} \mathrm{~N}_{2} \mathrm{O}_{4}$ : 1546.9); elemental analysis: calcd (\%) for $\mathrm{C}_{64} \mathrm{H}_{40} \mathrm{~F}_{34} \mathrm{~N}_{2} \mathrm{O}_{4}$ : C 49.69, $\mathrm{H}$ 2.61, N 1.81; found C 49.59, H 2.66, N 1.82; UV/Vis $\left(\mathrm{CHCl}_{3}\right): \lambda_{\max }(\varepsilon)=510(57220), 477 \mathrm{~nm}$ $\left(40412 \mathrm{M}^{-1} \mathrm{~cm}^{-1}\right)$; fluorescence $\left(\mathrm{CHCl}_{3}\right): \lambda_{\max }=526 \mathrm{~nm}$, fluorescence quantum yield $\Phi_{\mathrm{fl}}=0.91$; $\mathrm{CV}\left(\mathrm{CH}_{2} \mathrm{Cl}_{2}, 0.1 \mathrm{M}\right.$ TBAHFP, vs. $\left.\mathrm{Fc} / \mathrm{Fc}^{+}\right): \mathrm{E}_{1 / 2}(\mathrm{PBI} / \mathrm{PBI})^{-}=-0.67 \mathrm{~V}, \mathrm{E}_{1 / 2}\left(\mathrm{PBI}^{-} / \mathrm{PBI}^{2-}\right)=-0.99 \mathrm{~V}$.

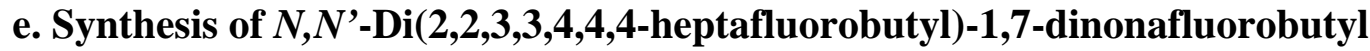 perylene-3,4:9,10-tetracarboxylic acid bisimide (2b).}

According to general procedure, the suspension of $700 \mathrm{mg} \mathrm{Cu}$ bronze $(10.9 \mathrm{mmol})$ in dry $20 \mathrm{ml}$ DMSO was heated at $125{ }^{\circ} \mathrm{C}$ for $15 \mathrm{~min}$ under nitrogen. $1.9 \mathrm{~g}$ Iodononafluorobutane $(5.5 \mathrm{mmol})$ was added dropwise. After $45 \mathrm{~min}$, a solution of $1 \mathrm{~g} \mathbf{1 b}(1.1 \mathrm{mmol})$ in $5 \mathrm{ml}$ DMSO was added dropwise, and the reaction mixture was stirred at $125{ }^{\circ} \mathrm{C}$ for $8 \mathrm{~h}$ yielded after column chromatography $\left(\mathrm{CH}_{2} \mathrm{Cl}_{2}: n\right.$-hexane $\left.=1: 2\right) 87 \%$ of $\mathbf{2} \mathbf{b}$ as an orange solid.

${ }^{1} \mathrm{H}$ NMR $\left(\mathrm{CDCl}_{3}, 400 \mathrm{MHz}, 298 \mathrm{~K}\right): \delta=8.99$ (s, 2H, perylene-H), 8.75 (d, 2H, J = $8 \mathrm{~Hz}$, perylene-H), 8.29 (s, 2H, perylene-H), 4.98 (t, 4H, J = 15.2 Hz, 2,2,3,3,4,4,4-heptafluorobutyl-H); ${ }^{13} \mathrm{C} \mathrm{NMR}\left(\mathrm{CDCl}_{3}, 100 \mathrm{MHz}, 298 \mathrm{~K}\right): \delta=162.2,162.1,135.9,132.8,132.0,131.6,128.4,128.2$, $127.4,127.1,126.9,122.9,122.5,119.1,118.7,117.4,116.6,116.2,115.9,115.1,114.8,114.5$, 114.0, 113.4, 112.2, 111.9, 111.5, 111.2, 109.2, 108.9, 108.6, 38.8, 38.6, 38.4. MS (MALDI-TOF): $\mathrm{m} / \mathrm{z}\left(\mathrm{M}^{-}\right)=1190.5$ (calcd for $\mathrm{C}_{40} \mathrm{H}_{10} \mathrm{~F}_{32} \mathrm{~N}_{2} \mathrm{O}_{4}$ : 1190.5); elemental analysis: calcd (\%) for $\mathrm{C}_{40} \mathrm{H}_{10} \mathrm{~F}_{32} \mathrm{~N}_{2} \mathrm{O}_{4}$ : C 40.36, H 0.85, N 2.35; found C 40.92, H 0.92, N 2.34; UV/Vis $\left(\mathrm{CHCl}_{3}\right): \lambda_{\max }(\varepsilon)$ $=506(60079), 474 \mathrm{~nm}\left(43410 \mathrm{M}^{-1} \mathrm{~cm}^{-1}\right)$; fluorescence $\left(\mathrm{CHCl}_{3}\right): \lambda_{\max }=528 \mathrm{~nm}$, fluorescence quantum yield $\Phi_{\mathrm{fl}}=0.94 ; \mathrm{CV}\left(\mathrm{CH}_{2} \mathrm{Cl}_{2}, 0.1 \mathrm{M}\right.$ TBAHFP, vs. $\left.\mathrm{Fc} / \mathrm{Fc}^{+}\right): \mathrm{E}_{1 / 2}(\mathrm{PBI} / \mathrm{PBI})=-0.60 \mathrm{~V}$, $\mathrm{E}_{1 / 2}\left(\mathrm{PBI}^{-} / \mathrm{PBI}^{2-}\right)=-0.88 \mathrm{~V}$.

\section{f. Synthesis of $N, N^{\prime}$-Di-n-butyl-1,7-dinonafluorobutylperylene-3,4:9,10- tetracarboxylic acid bisimide (2c).}

According to general procedure, the suspension of $970 \mathrm{mg} \mathrm{Cu}$ bronze $(15.2 \mathrm{mmol})$ in dry $20 \mathrm{ml}$ DMSO was heated at $125{ }^{\circ} \mathrm{C}$ for $15 \mathrm{~min}$ under nitrogen. $2.6 \mathrm{~g}$ Iodononafluorobutane $(7.5 \mathrm{mmol})$ 
was added dropwise. After $45 \mathrm{~min}$, a solution of $1 \mathrm{~g}$ 1c $(1.5 \mathrm{mmol})$ in $5 \mathrm{ml}$ DMSO was added dropwise, and the reaction mixture was stirred at $125{ }^{\circ} \mathrm{C}$ for $8 \mathrm{~h}$ yielded after column chromatography $\left(\mathrm{CH}_{2} \mathrm{Cl}_{2}: n\right.$-hexane $\left.=1: 2\right) 80 \%$ of $2 \mathbf{c}$ as an orange solid.

${ }^{1} \mathrm{H}$ NMR $\left(\mathrm{CDCl}_{3}, 400 \mathrm{MHz}, 298 \mathrm{~K}\right): \delta=8.92(\mathrm{~s}, 2 \mathrm{H}$, perylene-H), $8.68(\mathrm{~d}, 2 \mathrm{H}, \mathrm{J}=8 \mathrm{~Hz}$, perylene-H), 8.24 (s, 2H, perylene-H), 4.17 (t, $4 \mathrm{H}, \mathrm{J}=8 \mathrm{~Hz}, n$-butyl-H), 1.69 (quin, $4 \mathrm{H}, \mathrm{J}=8 \mathrm{~Hz}$, $\mathrm{J}=7.2 \mathrm{~Hz} n$-butyl-H), 1.37-1.46 (sext, $4 \mathrm{H}, \mathrm{J}=7.2 \mathrm{~Hz}, n$-butyl-H), 0.94 (t, $6 \mathrm{H}, \mathrm{J}=7.2 \mathrm{~Hz}$, $n$-butyl-H); ${ }^{13} \mathrm{C}$ NMR $\left(\mathrm{CDCl}_{3}, 100 \mathrm{MHz}, 298 \mathrm{~K}\right): \delta=162.4,135.4,132.3,131.2,130.9,128.3$, 128.0, 126.8, 126.6, 126.4, 123.7, 123.2, 119.4, 118.8, 116.8, 116.4, 116.3, 115.9, 115.6, 114.5, 114.2, 111.6, 109.2, 108.8, 40.7, 30.2, 20.3, 13.8. MS (MALDI-TOF): $\mathrm{m} / \mathrm{z}\left(\mathrm{M}^{-}\right)=938.4$ (calcd for $\mathrm{C}_{40} \mathrm{H}_{24} \mathrm{~F}_{18} \mathrm{~N}_{2} \mathrm{O}_{4}$ : 938.6); elemental analysis: calcd (\%) for $\mathrm{C}_{40} \mathrm{H}_{24} \mathrm{~F}_{18} \mathrm{~N}_{2} \mathrm{O}_{4}$ : C 51.19, H 2.58, $\mathrm{N} 2.98$; found C 51.35, H 2.62, N 2.94; UV/Vis $\left(\mathrm{CHCl}_{3}\right): \lambda_{\max }(\varepsilon)=508$ (49425), $476 \mathrm{~nm}\left(35285 \mathrm{M}^{-1} \mathrm{~cm}^{-1}\right)$; fluorescence $\left(\mathrm{CHCl}_{3}\right): \lambda_{\max }=528 \mathrm{~nm}$, fluorescence quantum yield $\Phi_{\mathrm{fl}}=0.97 ; \mathrm{CV}\left(\mathrm{CH}_{2} \mathrm{Cl}_{2}, 0.1 \mathrm{M}\right.$ TBAHFP, vs. $\left.\mathrm{Fc} / \mathrm{Fc}^{+}\right): \mathrm{E}_{1 / 2}(\mathrm{PBI} / \mathrm{PBI})=-0.72 \mathrm{~V}, \mathrm{E}_{1 / 2}\left(\mathrm{PBI}^{-} / \mathrm{PBI}^{2-}\right)=-0.98 \mathrm{~V}$.

\section{Molecular structure and Crystal packing}

\section{a. Molecular structure and Crystal packing of $\mathbf{2 b}$}

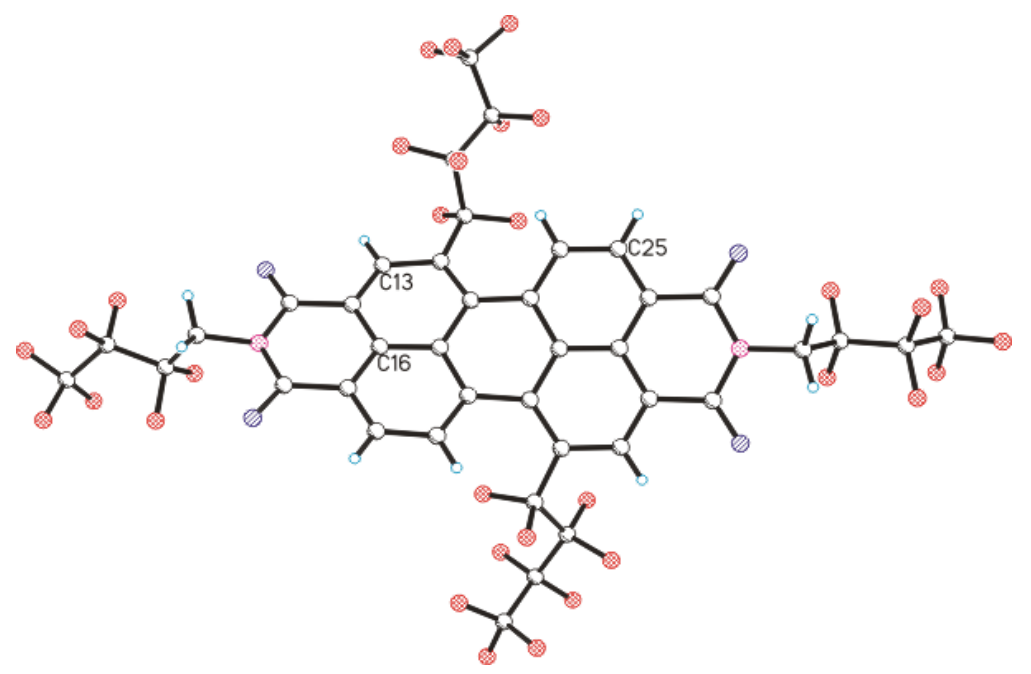

Figure S1. Molecular structure of 2b

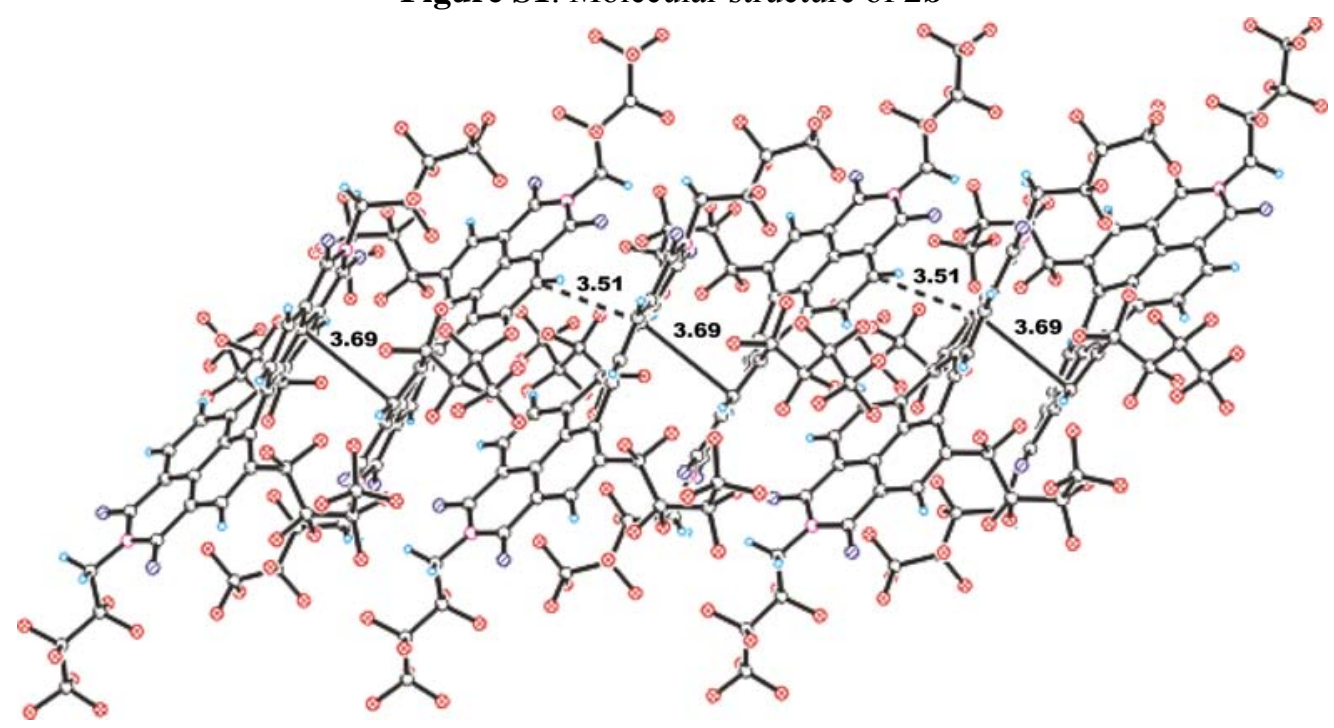

Figure S2. Crystal packing of $\mathbf{2 b}$ 


\section{b. Molecular structure and Crystal packing of 2c}

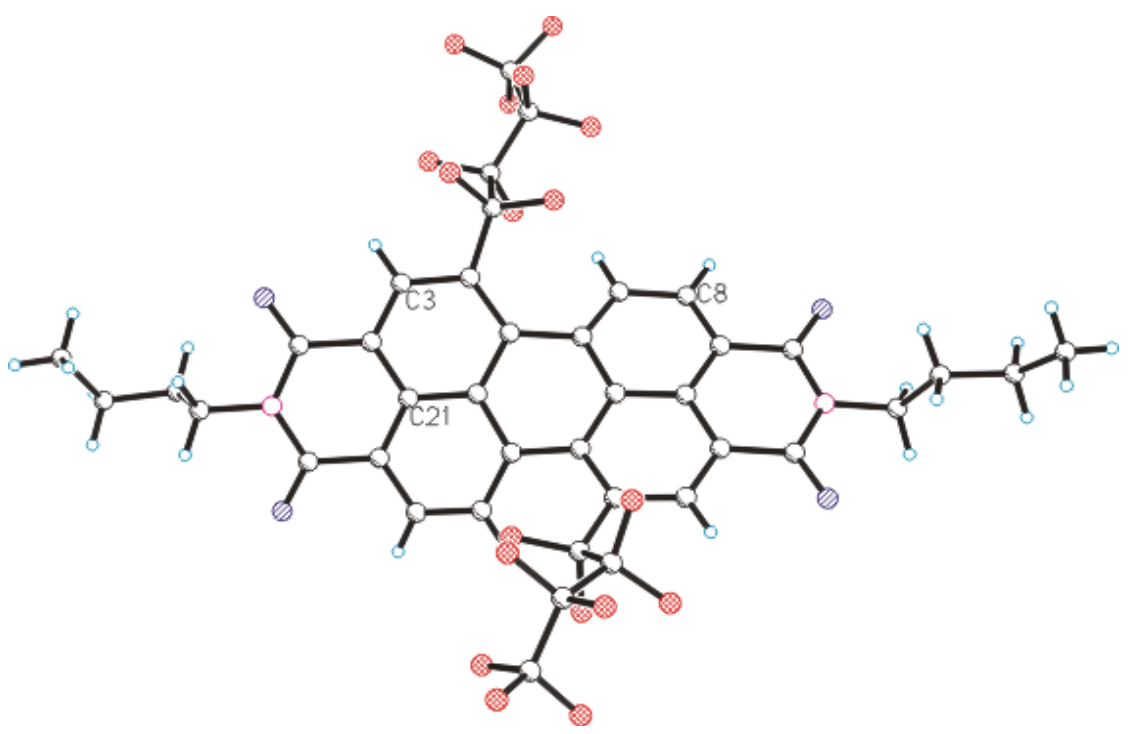

Figure S3. Molecular structure of 2c

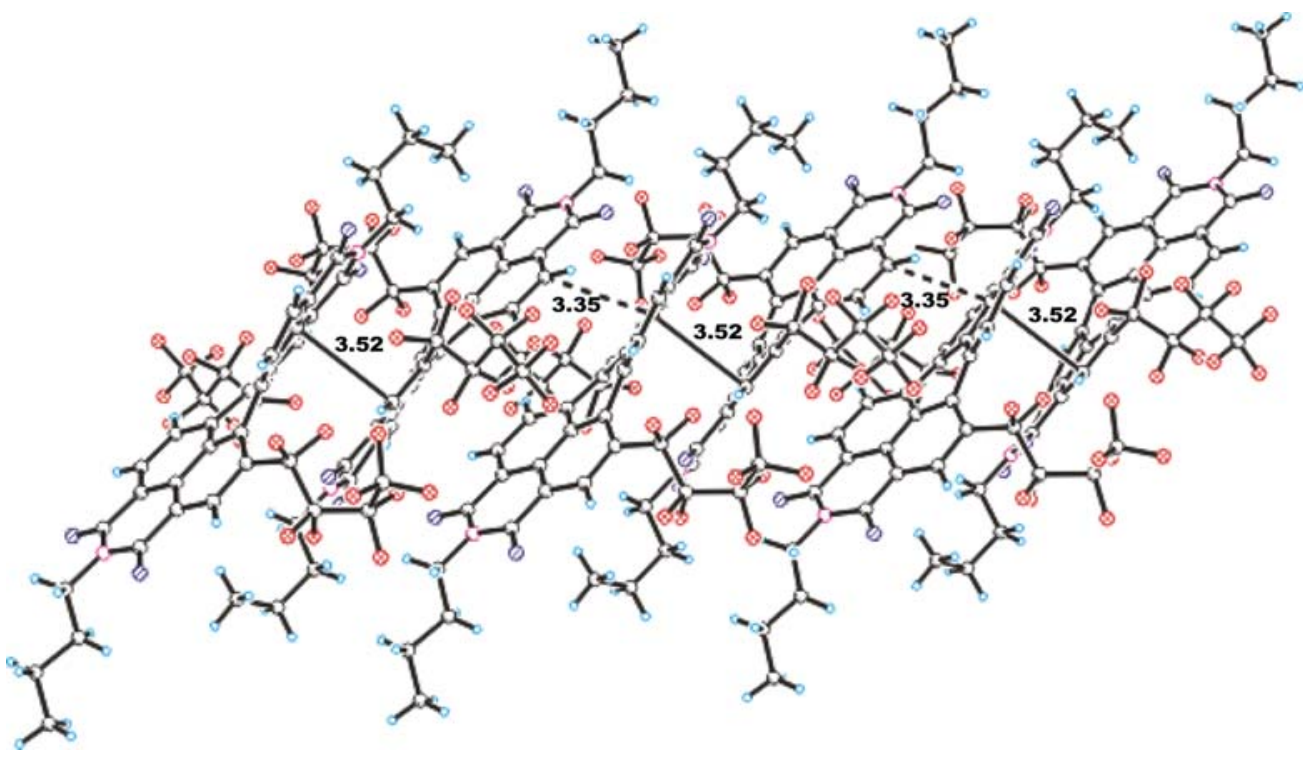

Figure S4. Crystal packing of 2c

The molecular structure and crystal packing of $\mathbf{2 b}$ and $\mathbf{2 c}$ are shown in Figure S1-S4. The stacked molecules share more than $50 \%$ of their core surface, between which the interplanar separation corresponds approximately to a distance of $3.69 \AA$ between $\mathrm{C} 13$ and $\mathrm{C} 16$ (solid line) for $\mathbf{2 b}$ and $3.52 \AA$ between $\mathrm{C} 3$ and $\mathrm{C} 21$ (solid line) for 2c. The closest intermolecular contact is of $3.51 \AA$ between $\mathrm{C} 16$ and $\mathrm{C} 25$ (dash line) for $\mathbf{2 b}$ and $3.35 \AA$ between $\mathrm{C} 8$ and $\mathrm{C} 21$ (dash line) for 2c respectively. 


\section{Referrences:}

(1) Lakowicz J. R. Principles of Fluorescence Spectroscopy, 2nd ed., Kluwer Academic/Plenum, New York, 1999, 52.

(2) Seybold, G.; Wagenblast, G. Dyes Pigm. 1989, 11, 303.

(3) For the synthesis of 1a-c, see: (a) Rohr, U.; Kohl, C.; Müllen, K.; van de Craats, A.; Warman, J. J. Mater. Chem 2001, 11, 1789.(b) Jones, B.A.; Marks, T.J.; Wasielewski, M.R. Angew Chem. Int. Ed. 2004, 43, 6363. (c) Böhm, A.; Arms, H.;Henning, G.; Blaschka, P. (BASF AG) German Pat. DE 19547209 A1, 1997; Chem. Abst. 1997, 127, 96569g. 
${ }^{1} \mathrm{H}$ NMR spectrum of $\mathbf{2 a}$ in $\mathrm{CDCl}_{3}$

$\odot \odot 0^{\circ} \odot$

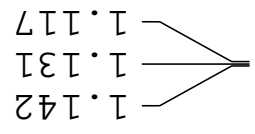

$\angle S 9^{\circ} Z$ $\varepsilon \angle 9^{\circ} 乙$ $069^{\prime} z$ 9०L'乙 $\varepsilon 乙 L ' 乙-$<smiles>[10BH2-]</smiles>

662 ' $L$ 6IE' $L$

9†t' $L$

S9t ' $L$

†8 ' $L$

๑E⿷ ' 8

ISL ' 8 OLL' 8 โ86. 8

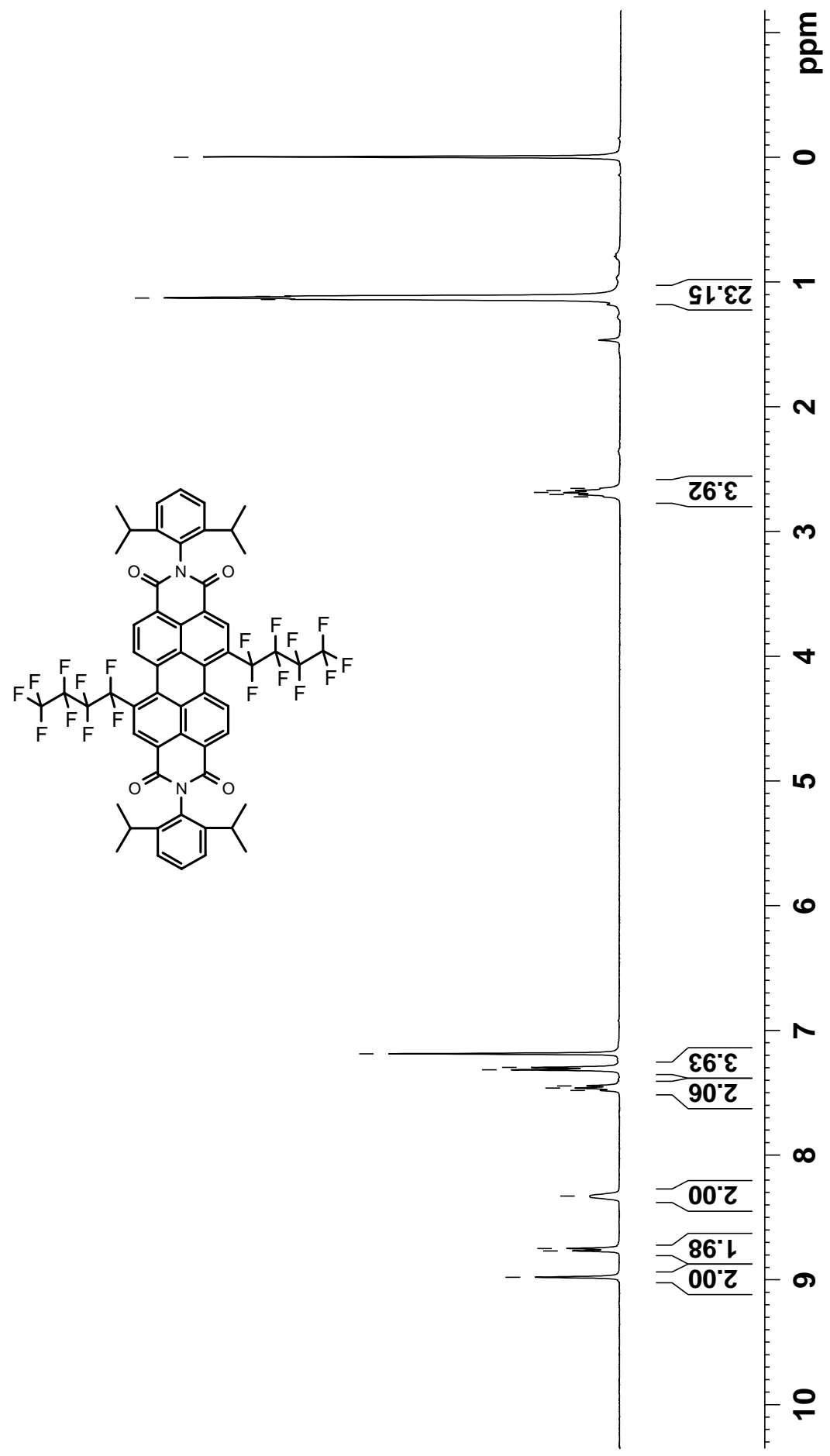


${ }^{13} \mathrm{C}$ NMR spectrum of 2a in $\mathrm{CDCl}_{3}$

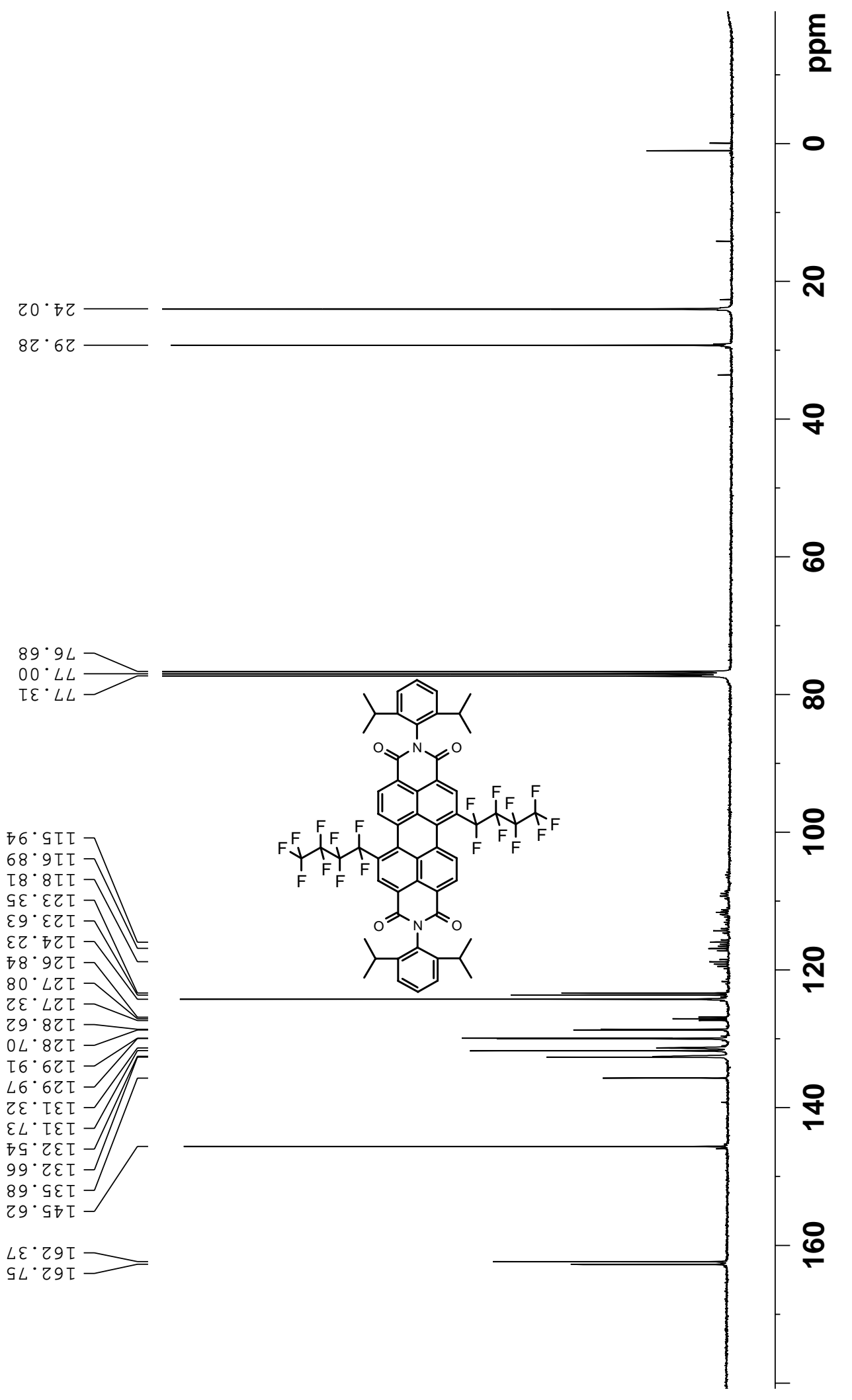


${ }^{1} \mathrm{H}$ NMR spectrum of $3 a$ in $\mathrm{CDCl}_{3}$

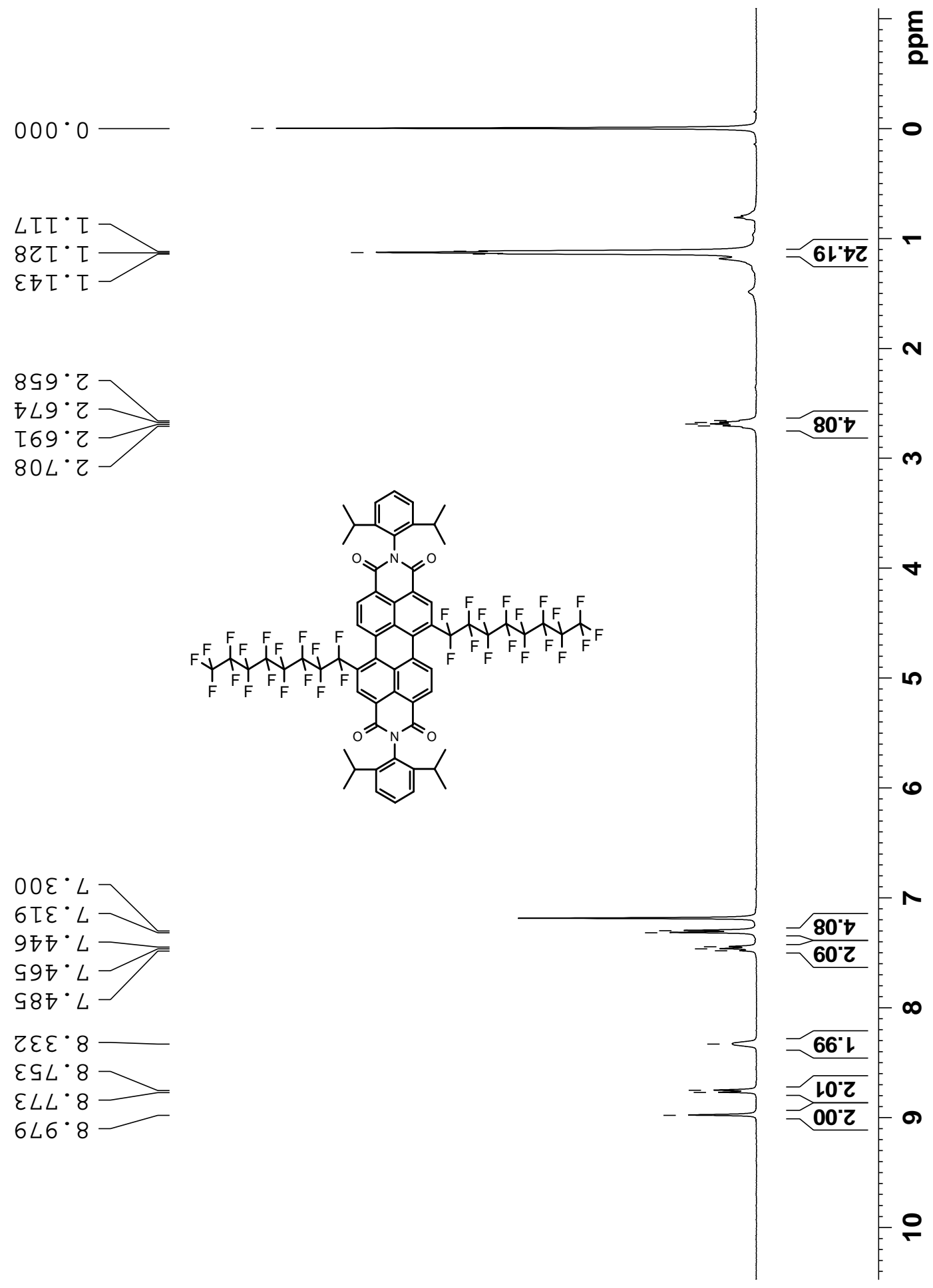


${ }^{13} \mathrm{C}$ NMR spectrum of $\mathbf{3 a}$ in $\mathrm{CDCl}_{3}$

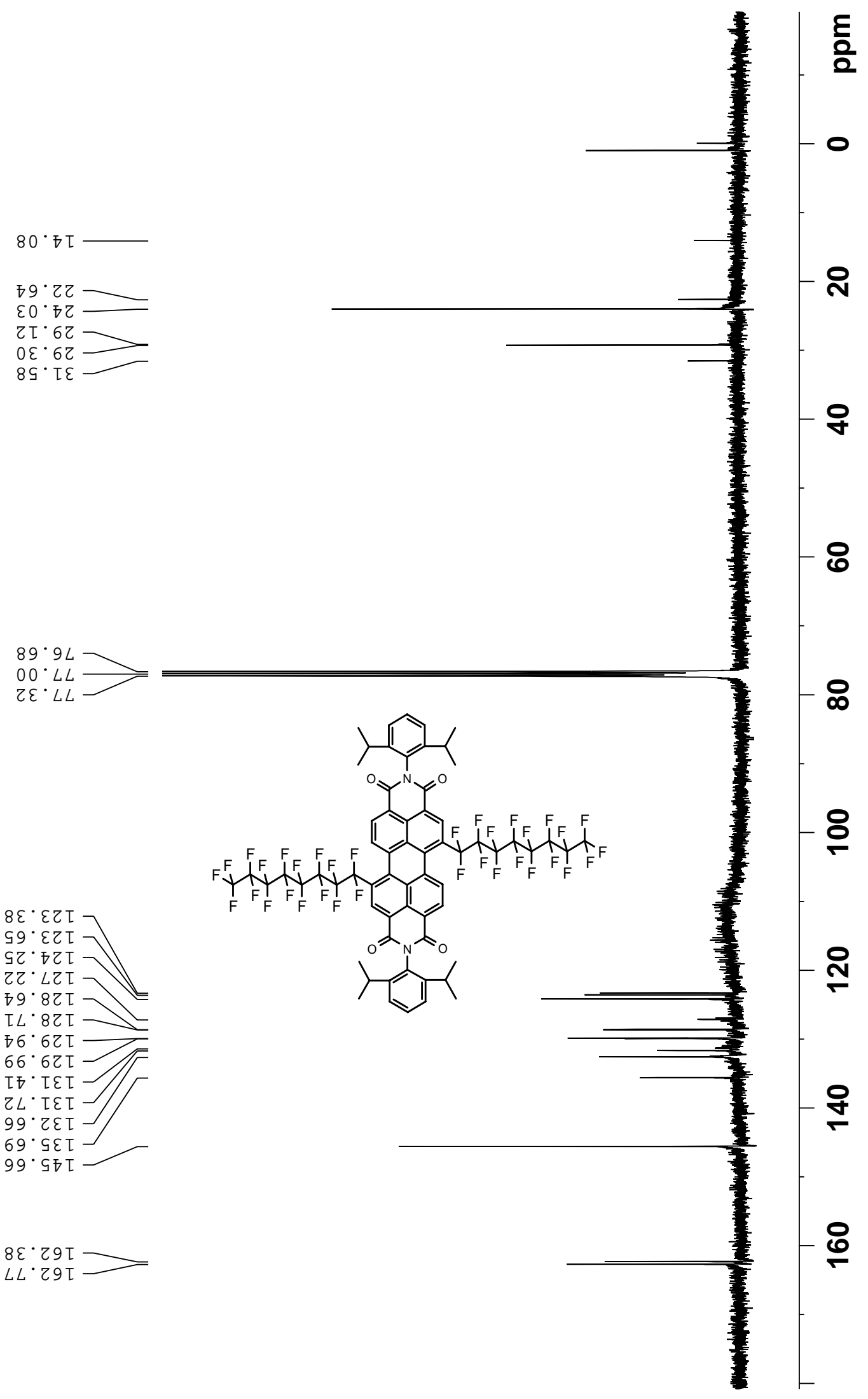


${ }^{1} \mathrm{H}$ NMR spectrum of $\mathbf{2} \mathbf{b}$ in $\mathrm{CDCl}_{3}$

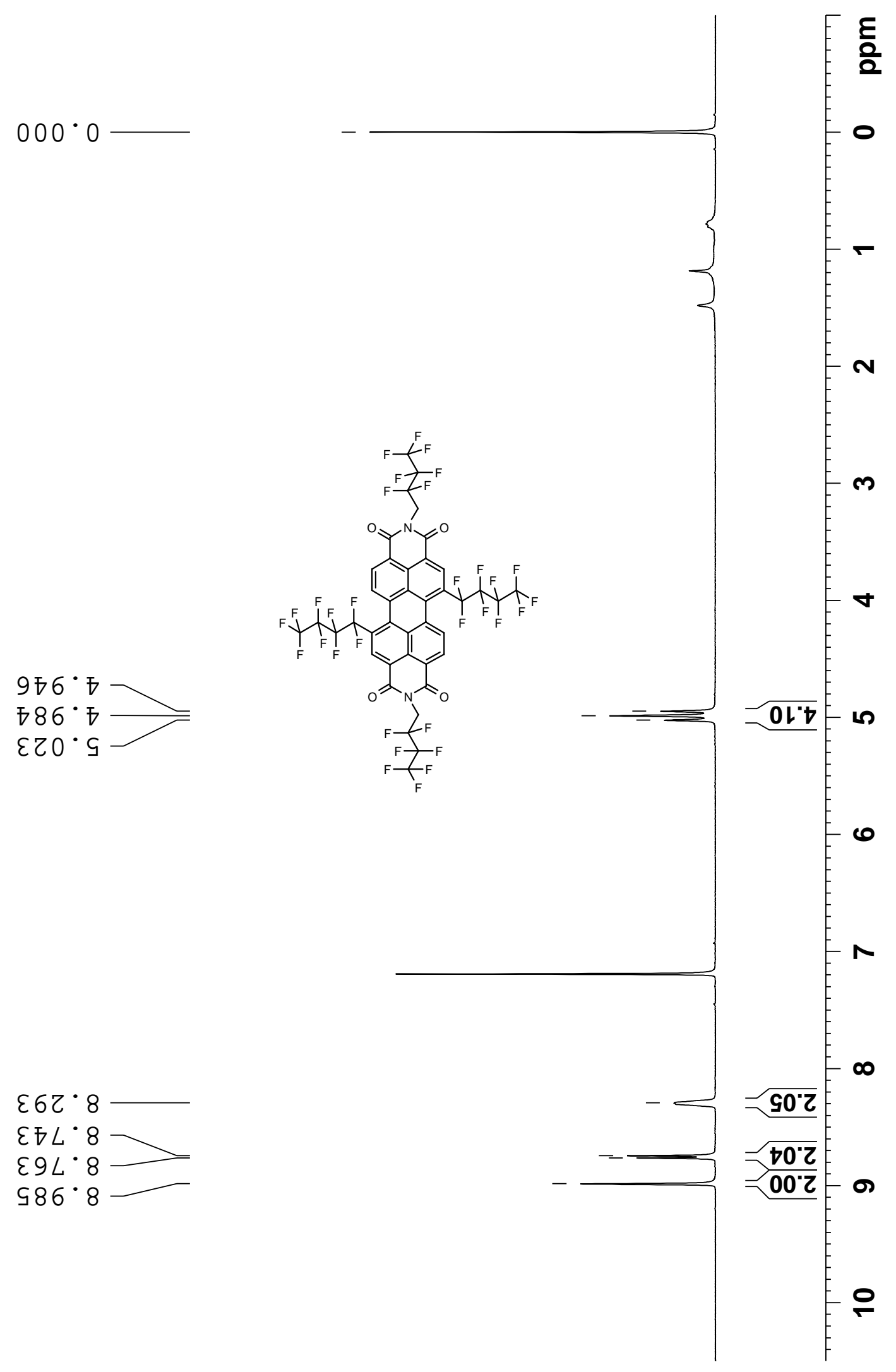


${ }^{13} \mathrm{C}$ NMR spectrum of $\mathbf{2 b}$ in $\mathrm{CDCl}_{3}$
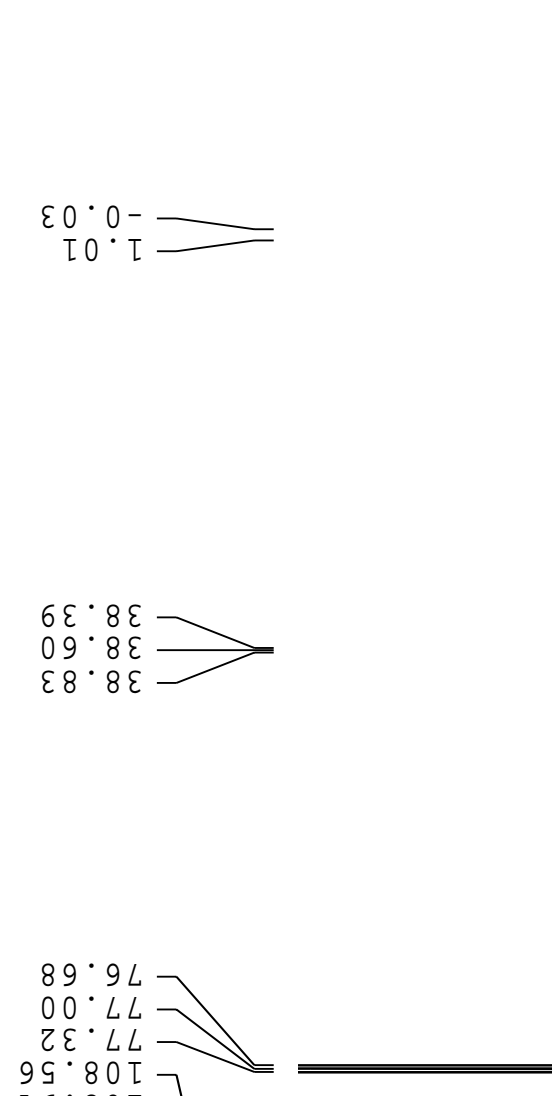
$\rightarrow 6.80[]$ 6 . $60 \mathrm{~T}$ SI. III E9' [IL] $\downarrow 乙 \cdot Z \tau$ $9 \varepsilon \cdot \varepsilon[I-$ $20^{\circ} \circ[I]$ IG' $\rightarrow I$ हI. $9 \tau$ $88.9 \tau$ IZ'9II I9. 9 II $O D^{\circ} \angle I I$ $\checkmark L: 8 I I$ $\angle O 0^{\circ} 6$ I \&9'ZZI ह6. ZZL 88'9ZI ZI' $\angle Z I$ $9 \varepsilon$ ' $L Z T$ GI.8ZT Et' $82 I$ $09^{\circ}$ IEI $66^{\circ}$ TEI $6 L$ 'ZEL G8. $9 \varepsilon \tau$ $90.29 I$

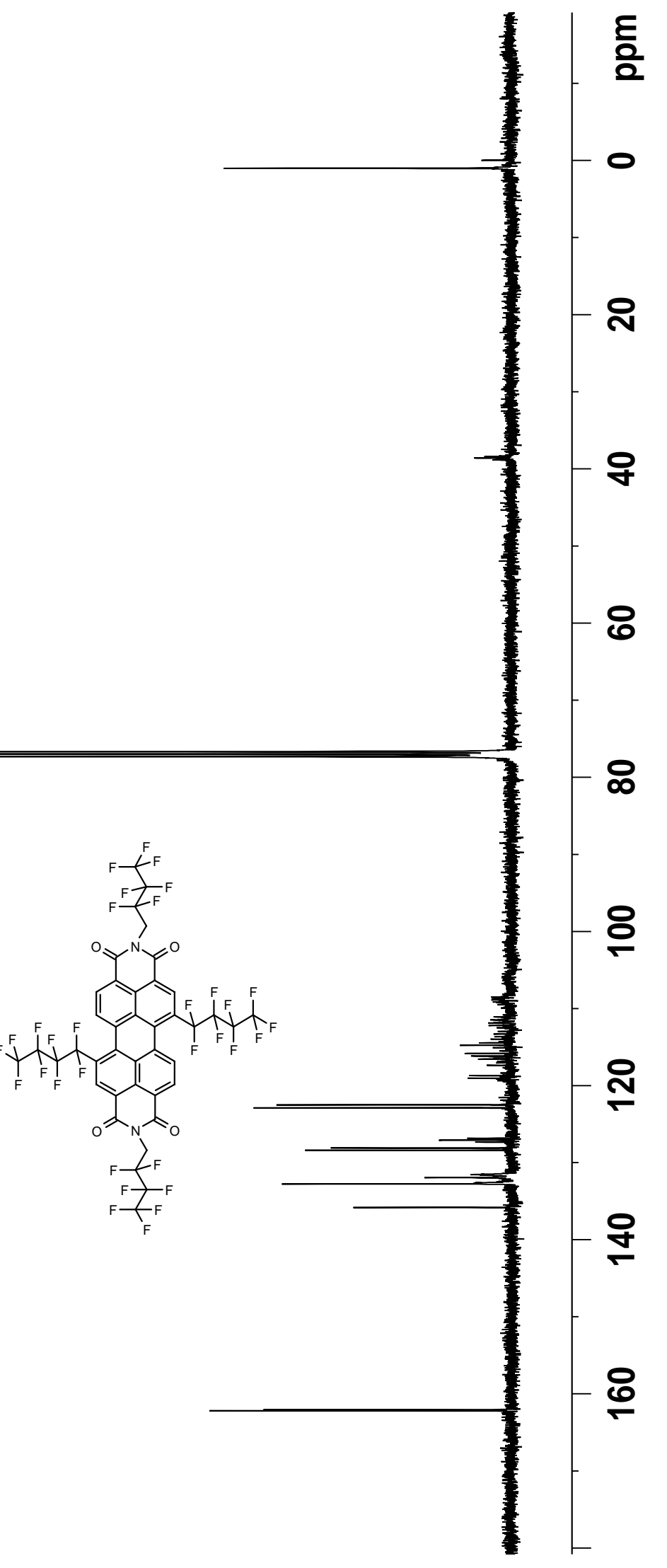


${ }^{1} \mathrm{H}$ NMR spectrum of $\mathbf{2 c}$ in $\mathrm{CDCl}_{3}$

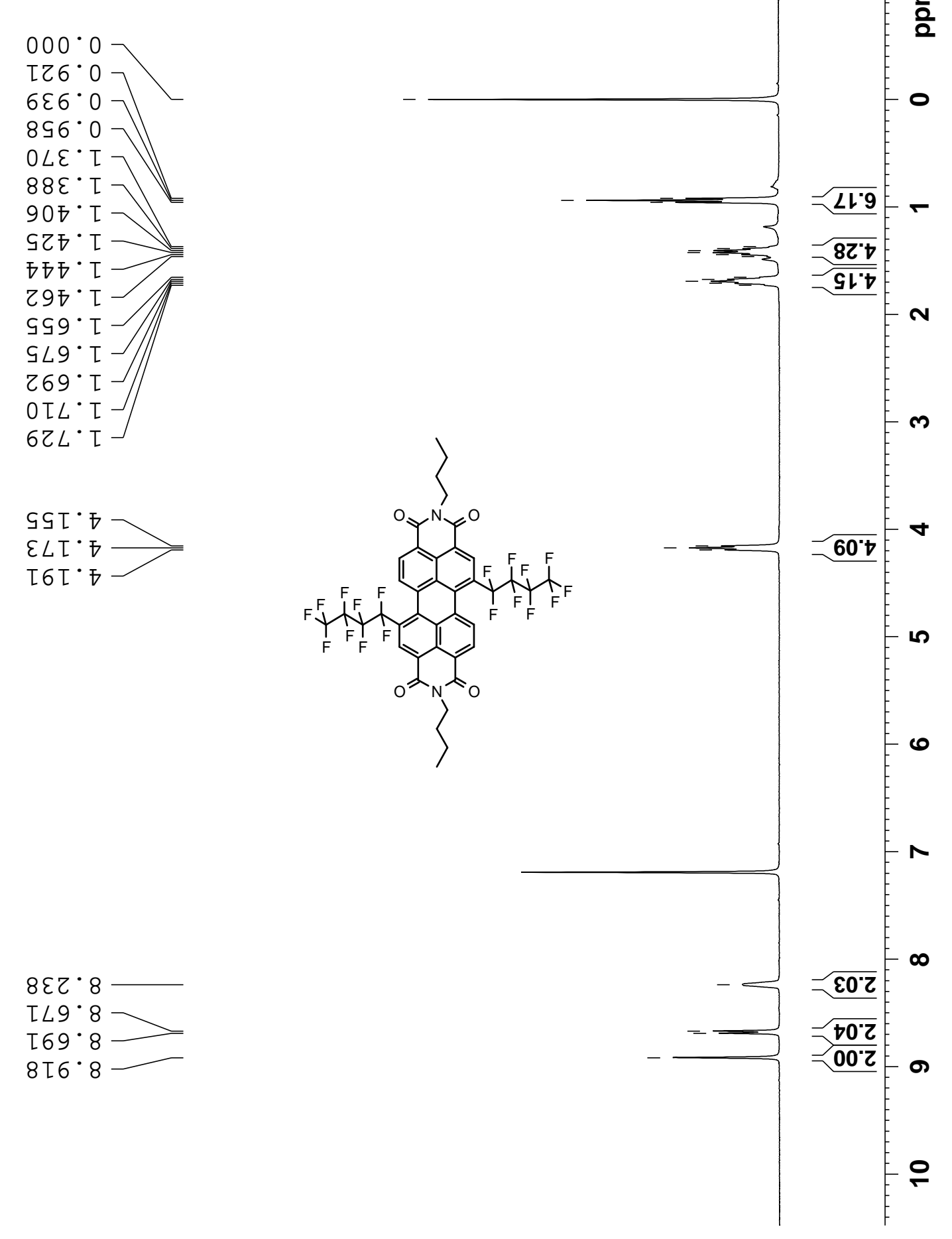


${ }^{13} \mathrm{C}$ NMR spectrum of $2 \mathrm{c}$ in $\mathrm{CDCl}_{3}$

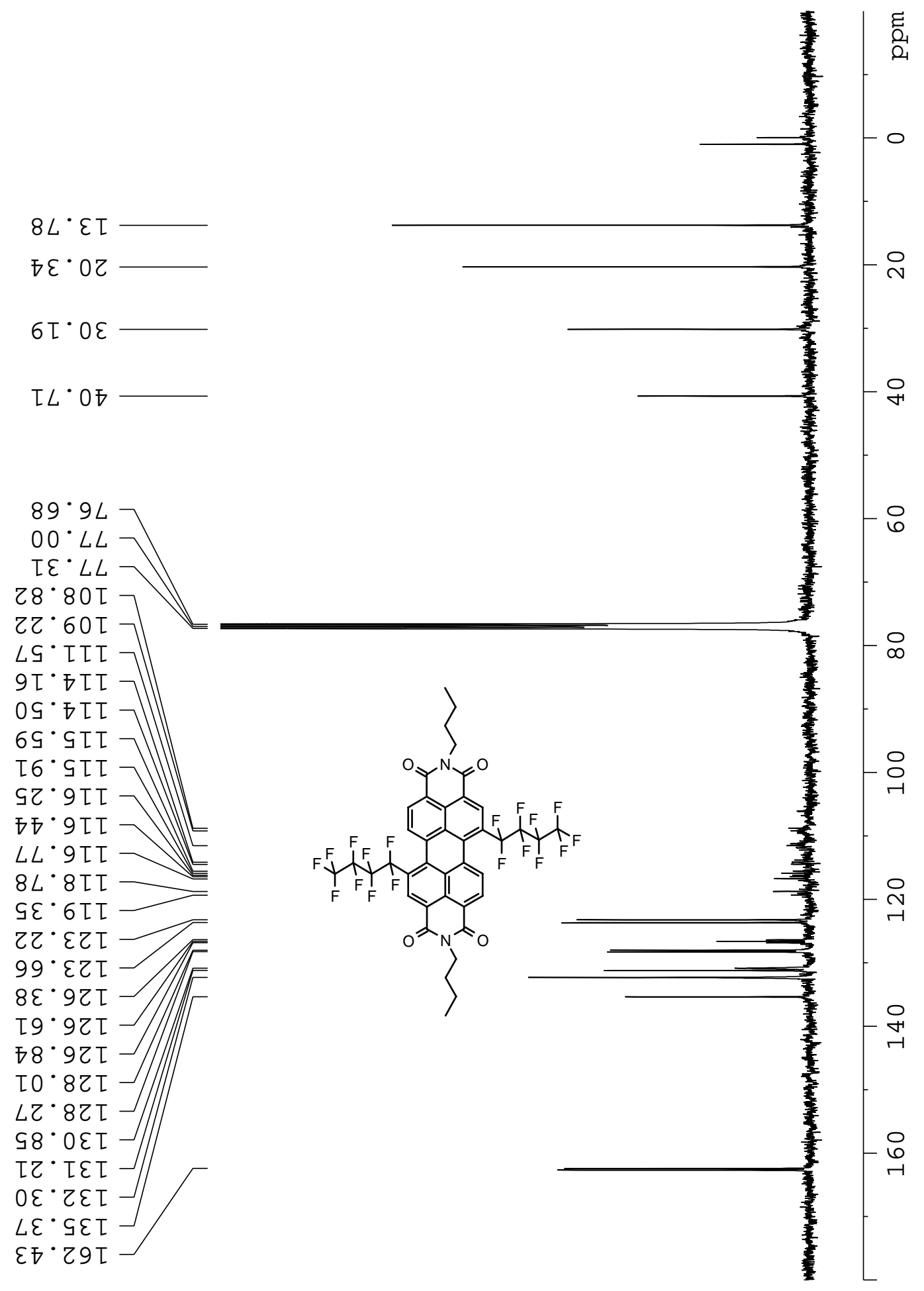

